\title{
Trinetra: a solution to handle cross-VM time-driven attack
}

\author{
Dhara H. Buch ${ }^{1} \cdot$ Haresh S. Bhatt ${ }^{2}$
}

Received: 31 October 2019 / Accepted: 18 February 2020 / Published online: 2 March 2020

(c) Springer Nature Switzerland AG 2020

\begin{abstract}
The efficient utilization of hardware and software resources plays a vital role in a high-performance computing environment. Where on the one side, a shared pool of resources facilitates faster processing with limited resources, this mechanism also widens the scope of many kinds of security attacks on the other side. Side-channel attack (SCA) is one such attack where methods to monitor the activity of exploited shared resource is carried out to extract the private key. One such SCA, branch prediction analysis attack, launched with time-driven attack (TDA) method is considered in this paper. With the consideration of the virtualization environment, proposal of an algorithm Trinetra, to detect the presence of cross-VM TDA, is the primary focus of our paper. We have tested the performance of Trinetra with experimental analysis in addition to the attack simulation. Performance evaluation has found the Trinetra very effective with negligible performance overhead.
\end{abstract}

Keywords Side-channel attack · Branch prediction analysis attack · Time-driven attack · Virtualization

\section{Introduction}

Virtualization hides the internal details of the underlying resources from the end users. Even though operated by the same host, co-hosted virtual machines (VMs) are separated from one another by virtual machine manager (VMM) with the property of isolation. However, the pool of resources shared among the co-hosted VMs raises many vulnerability issues, one of them is the covert channel. A covert channel is a method of communication that is used to illicitly transfer information via shared resources, thus breaking the security policy of the system [1]. Side-channel attack (SCA) is one such attack that exploits the shared physical resources like CPU cache, a memory bus, disk bus, branch target buffer (BTB) and network queue. Co-resident virtual machines use a covert channel to leak confidential information [2].

Cloud administrator can configure the VMs in three ways: (a) fully isolated, (b) fully shared and (c) hybrid. First provides configuration which is similar to that of dedicated machine. It provides high security, but lacks in terms of effective resource utilization. Second is used for best resource utilization, by means of resource sharing, while it lacks in terms of security. The third is a defacto standard for efficient resource management with optimized security. We have taken the defacto standard of the VM configuration for our study.

Ristenpart et al. [3]. have shown a possibility of placing a VM co-resident to the target VM by careful empirical mapping with Amazon's EC2 as the case study. Additionally, in a virtual network, services running on a VM like Hypertext Transfer Protocol Secure, Secure File Transfer Protocol, Simple Mail Transfer Protocol Service or Proxy service can also be exploited by an attacker to attack a co-resident victim VM(s). An attacker or compromised VM can exploit the shared resources to launch SCA on the other co-resident VM giving rise to cross-VM SCA. The SCA, which exploits the components of branch prediction unit like branch target buffer and branch predictor, is known as the branch prediction analysis attack.

Dhara H. Buch, dhara_buch020@gtu.edu.in; Haresh S. Bhatt, haresh@sac.isro.gov.in | 'Gujarat Technological University, Ahmedabad, Gujarat 382424, India. ${ }^{2}$ Information \& Network Security, Space Application Centre, ISRO, Ahmedabad, India. 
BPA attack is meant to extract asymmetric private key of the public-private asymmetric cryptographic algorithms like RSA (Rivest-Shamir-Adleman) and ECC (elliptic-curve cryptography). Algorithms like RSA and ECC use squareand-multiply algorithm that contains a conditional branch instruction for which status of execution depends on the corresponding bit of the private key. Targeting this conditional branch instruction, the attack was originally discussed by Onur [2], where four different methods to launch the attack were suggested. Onur et al. [2]. have considered a non-virtualized environment where they have stated that the attack is possible even in the presence of virtualization and sandboxing.

One of the total four suggested ways is called direct timing attack (DTA). DTA performs simulations over known data sets to monitor the behavior of the target process. The output of branch predictor is compared with the actual behavior of the conditional branch instruction for some known bits of the secret key for a large number of messages. The value of the next secret bit is predicted from the comparative results, regarding several mispredicted branches.

Among the other three suggested ways of launching a BPA attack in [2], two approaches suggest frequent clearance of the BTB which is either synchronized or asynchronized with the target process. The final method, timedriven method [4], recommends filling BTB with many branch instructions instead of clearing BTB. Clearance or filling entire or targeted positions of BTB forces the target process to call the conditional branch instruction from memory every time it is encountered, as its address from BTB is evicted. Hence, the execution time of this conditional instruction increases and the increased execution time implicitly signals the presence of key bit 1 for that position.

The study of the BPA attack revealed that there is a need to consider it in virtualization by taking the underlying configuration into account [5-7]. Additionally, we also need to study the applicability of the existing approaches for handling BPA attacks in the virtualization environment.
In [8], we have analyzed the scope of the BPA attack in the virtualization environment. We have discussed the cross-VM BPA attack, where one compromised VM launches a BPA attack on the other co-resident VM. Depending on the configuration, two VMs do or do not share the CPU core and cryptographic library. Accordingly, the applicability of different attack launching methodologies in possible attack environments is also discussed in the same. Table 1 presents the summary of the discussion, which reveals that the BPA attack can be launched by any of the three methods other than DTA if the CPU core is shared, but the cryptographic library is not shared. If the two VMs have a separate core, then with only the DTA method cross-VM BPA attack is possible provided the cryptographic library is shared.

The study of existing work done in handling BPA attacks [9-12] reveals that there is a need to propose a new approach to overcoming the limitations of existing ones. Although BPA attack can be launched by four different methods, we have focused on the time-driven attack method in this paper. We have proposed an approach Trinetra to detect cross-VM time-driven attack.

The rest of the paper is organized as follows: Sect. 2 provides the background details. Section 3 presents the goal and objectives of this paper. Further, the related work is discussed in Sect. 4. A brief overview of time-driven attack methodology is given in Sect. 5. In Sect. 6, we have explained the proposed algorithm Trinetra. Section 7 discusses the results obtained for setting the threshold values for the attack detection. Attack simulation and experimental analysis are discussed in Sects. 8 and 9, respectively. Finally, the conclusion is given in Sect. 10.

\section{Background}

In the current era, the cloud has become a defacto standard for efficient resource utilization over the Internet. Cloud uses virtualization technology for providing on the fly on demand required resources to end users.

Table 1 Attack environments for BPA attack in virtualization [8]

\begin{tabular}{|c|c|c|c|c|}
\hline \multirow[t]{2}{*}{ BP attack launching mechanism } & \multicolumn{4}{|c|}{ Target and compromised processes on the different VMs } \\
\hline & $\begin{array}{l}\text { VMs do not share library } \\
\text { and CPU core }\end{array}$ & $\begin{array}{l}\text { VMs share library } \\
\text { and CPU core }\end{array}$ & $\begin{array}{l}\text { VMs share library, but do } \\
\text { not share CPU core }\end{array}$ & $\begin{array}{l}\text { VMs do not share a } \\
\text { library, but share CPU } \\
\text { core }\end{array}$ \\
\hline Direct timing attack & Not possible & Possible & Possible & Not possible \\
\hline Asynchronous attack & Not possible & Possible & Not possible & Possible \\
\hline Synchronous attack & Not possible & Possible & Not possible & Possible \\
\hline Time-driven attack & Not possible & Possible & Not possible & Possible \\
\hline
\end{tabular}


Internal detail of the host system is kept hidden from the end user as per the concept of virtualization. The cloud computing environment is comprised of a physically scattered but an integrated pool of resources. The demanded hardware and software resources are grouped to form a logical unit and presented to the user in the form of a virtual machine (VM). All the VMs run independently to each other with required isolation. In the virtualization layer, a virtual machine monitor (VMM) or hypervisor is the core entity that manages and controls the VMs. It is the VMM that provides isolation among VMs for the means of providing secure services. A VMM is installed on the host system, where it can be categorized as a bare-metal or hosted type based on its operating capability. A VMM can manage multiple VMs, where the resources of the underlying host system are shared among the VMs. VMs operating on the same host, managed by the same hypervisor, operating on the same host, are known as co-hosted or co-resident VMs.

Virtualization plays an important role in imposing security by preventing users from directly accessing resources. However, there are many kinds of security issues that may raise even in the presence of virtualization. We have discussed them in [13], where one of the severe attacks is side-channel attack (SCA) which is launched by exploiting the shared resources.

A compromised VM can exploit the shared resources to launch an attack on the other co-resident $\mathrm{VM}$, resulting in a cross-VM SCA. Branch prediction analysis (BPA) attack is a type of SCA where the component of the branch prediction unit is exploited to launch the attack. A type of BPA attack, time-driven attack on cross-VM platform, is the main target of this paper.

\section{Goal and objectives}

Our paper focuses on the following objectives for timedriven attack in the virtualization environment: (1) proposal of a new solution, Trinetra, that can handle the crossVM TDA attack by overcoming the limitations of existing ones and (2) experimental analysis of the proposed approach for performance evaluation.

\section{Related work}

Branch prediction analysis attack was proposed by Onur et al. [2] where the square-and-multiply algorithm was exploited to predict the secret bits of the private key. Further, they themselves suggested solutions to prevent BPA attack by replacing the S\&M algorithm by Montgomery ladder [14] algorithm. However, S. Bhattacharya et al. have shown the possibility of a BPA attack even if the ladder algorithm is used for RSA implementation $[15,16]$. They have also shown that the BPA attack is possible even for RSA with Chinese remainder theorem (CRT) implementation [17].

For handling BPA attack, many approaches have been suggested. As one of the mitigation techniques, Agosta et al. [9] suggested modifying the code of crypto algorithms by eliminating the conditional instruction or by replacing them with balanced branch instructions. We need to implement this solution in each of the vulnerable algorithms.

Ya Tan et al. [10] have proposed an approach for preventing BPA attack launched with time-driven attack methodology. As per the suggested approach, BTB entries are kept locked for the process with high occupancy. Locking of BTB entries prevents spy process from occupying all the positions in BTB which are used by the target crypto algorithm. Hence, the spy process fails to predict the secret bits. However, the approach may result in a false positive. A blacklisting approach proposed by Julien et al. [11] allows only white-listed processes to access the performance counters. The suggested access control method works efficiently except the case where white-listed processes get compromised. In another BPA attack mitigation approach, S. Bhattacharya et al. [12] have targeted DTA methodology for which success relies on the behavior of the branch predictor. The suggested approach alters the state of the predictor by initiating a randomized module to make the spy process lose its pace in predicting secret bits.

An approach called SpyDetector [18] is an anomalybased supervised approach, which can detect the ongoing side-channel attack. Attacks typically carried out by creating intentional contentions in shared resources are handled by SpyDetector. Although only four attack types targeting cache is considered by SpyDetector, it can be extended to detect other types of SCA like BPA attack as discussed in the paper.

Among all the above-discussed methods, the only approach suggested by Julien et al. [11] considers virtualization as the underlying environment. However, other approaches can also be applied to handle BPA attack in virtualization. Although existing solutions can handle BPA attacks in virtualization, manipulation of architectural components like BTB [10], branch predictor [12] and performance counter [11] by them may affect the performance of the benign processes also.

The proposed solution Trinetra handles BPA attack considering virtualization as the underlying environment. Trinetra detects the presence of cross-VM TDA without affecting the performance of legitimate processes. Although we have focused on time-driven attack as an attack launching method, the approach can be extended to include other methods of launching a BPA attack also. The following 
section gives a brief overview of the time-driven attack procedure.

\section{Time-driven attack}

Onur et al. [2] discuss a time-driven BPA attack that is launched by a spy process in concurrence to a cryptographic process, i.e., RSA running on the same machine. As we consider cross-VM TDA in this paper, the victim process calling the RSA algorithm and the spy process is assumed to be present on separate, but co-resident VMs. Details of the actual attack procedure for cross-VM TDA are as follows:

- Continuously running the spy process fills all the entries of the BTB by executing a carefully chosen large number of conditional branch instructions.

- Conditional branch instruction (step 5 of Fig. 1) of the square-and-multiply (S\&M) algorithm called for exponential multiplication during the RSA decryption process is the target for launching TDA.

- When this conditional branch instruction of at step 5 is called, one of the BTB entries holding the target address of spy program instruction is required to be evicted to make room for the target conditional branch instruction of the RSA process.

- The spy process also measures the execution time after each pass in addition to keeping BTB continuously filled. The time gets affected when the conditional branch instruction is called because it results in swap in and swap out of one of the spy process instructions. An increase in execution time represents key bit 1 in the private key.

- The process is repeated until all the key bits are predicted.
Input:
C: Ciphertest
d: Private Key
n: Module
SqandMul (C, d,n)
$1 \quad \mathrm{M}^{\prime} \leftarrow 1$
2 For $\mathrm{i}=1$ to keylength
$3 \quad \mathrm{M}^{\prime}=\operatorname{MontMul}\left(\mathrm{M}^{\prime}, \mathrm{M}^{\prime}, \mathrm{n}\right)$;
4 If $\mathrm{di}=1$ then
$5 \quad \mathrm{M}^{\prime}=\operatorname{MontMul}\left(\mathrm{M}^{\prime}, \mathrm{C}, \mathrm{n}\right)$

Fig. 1 Square-and-multiply algorithm
For filling the BTB by conditional branch instructions, the spy process can apply either of the following approaches:

- Total occupancy: entire BTB is filled

- Partial occupancy: part of BTB where RSA instructions are associated is filled which is quite difficult to be implemented

In either of the above case, the execution time of the spy process is certainly affected by the execution of the conditional branch instruction of RSA.

With the consideration of the underlying attack procedure of time-driven attack, we hereby propose an algorithm to handle cross-VM TDA. The approach is discussed in the next section.

\section{Trinetra}

The procedure carried out by the spy process to launch time-driven attack employs the following primary actions:

- Filling of BTB entries

- Measurement of execution time

Measurement of execution time in the above action requires reading of hardware performance counters (HPCs) by generating an interrupt. A spy process of TDA performs the above two actions with very high frequency. Hence, the observation of the above two actions is very important for the detection point of view. However, a process cannot be declared as malicious just because of the presence of these actions as legitimate processes like application profiler and update manager may also perform them. Hence, the frequency of actions becomes an important parameter to differentiate a spy and a legitimate process. A process filling BTB and reading HPCs with high frequency can be certainly considered a spy process. However, it becomes very important to check the means behind the performed actions of the process for assured detection of TDA and that needs to dive deep into the implications of timedriven attack.

The attacker extracts the asymmetric private key (APK) of the RSA decryption process by launching TDA. Once the private key is available, the symmetric public key (SPK) which is encrypted using asymmetric private key will also be available to the attacker. Further, the attacker will be able to decrypt all the confidential messages that are encrypted using that SPK. However, the attacker must be successful in trapping packets holding encrypted SPK as well as all the data packets for launching a fruitful TDA. Thus, an obvious step that the attacker takes after 
launching the TDA is to trap the confidential data packets. Summarizing this, filling BTB and reading HPCs continuously followed by packet trapping are the three primary actions initiated by the compromised VM.

The proposed detection approach Trinetra observes the above-mentioned actions to detect the VM launching TDA. As represented in Fig. 2, Trinetra is comprised of three monitors, namely BTBAccess monitor (BM), interrupt monitor (IM) and network monitor (NM) to monitor the above-discussed three primary actions, respectively. The three components can be taken as three eyes, where BM and IM together can efficiently detect the presence of a VM generating TDA, and NM is an additional component launched for strengthening the detection process. Accordingly, an advanced trigger is sent to the computer emergency response team (CERT) when both BM and IM generates alert for a VM. Further, NM confirms the malicious intentions of that VM and sends the final trigger to CERT.

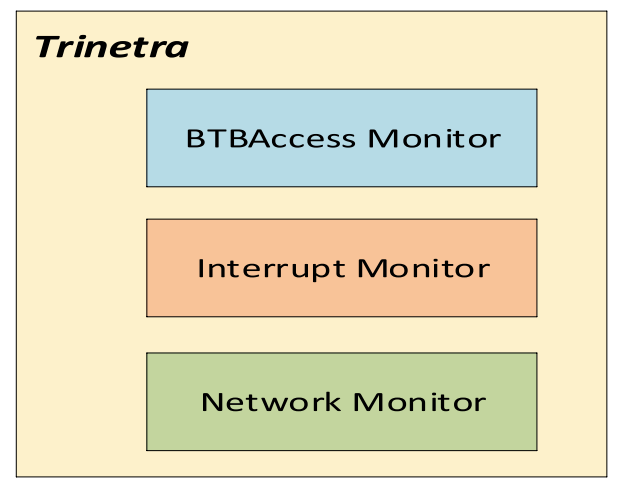

Fig. 2 Components of Trinetra
The attacker will attempt to access confidential data packets once the private key is extracted. Hence, monitoring of the packet trapping action is significant for strengthening the detection of the attacker. The third eye, NM, is initiated as a supporting component that assures the malicious intentions of the compromised VM for which both $\mathrm{BM}$ and IM generate an alert. The model and flow of Trinetra are presented in Figs. 3 and 4, respectively.

As shown in Fig. 4, both BM and IM are initiated simultaneously with initial status trustworthy for all the running VMs. If BM and/or IM generates alert for a VM, then its status is recommended as suspicious by either/both of the monitors individually. If a VM is launching TDA, then both $B M$ and IM will generate alert for that VM. In this case, both $B M$ and IM will individually recommend the status change to suspicious for the TDA launching VM. The status of such VM is changed to spy. Further, NM is turned on only for the spy VM, and when the NM generates alert for the spy VM, the status of the VM is changed to malicious. Detailed functional description of all the three monitors is given below:

(a) BTBAccess monitor: It monitors the frequency of BTB access, i.e., the number of times a process accesses BTB for executing conditional branch instructions. BTBAccess monitor module runs in the background on the host system. BTB occupancy of different processes is calculated by observing their BTB hits. The values of this event can be obtained by reading the appropriate model-specific register (MSR) by executing $r d m s r$ assembly language instruction of $\times 86$ architecture. We need to initialize ECX, hardware statistics counter and index registers with appropriate values for retrieving the details regarding the branch

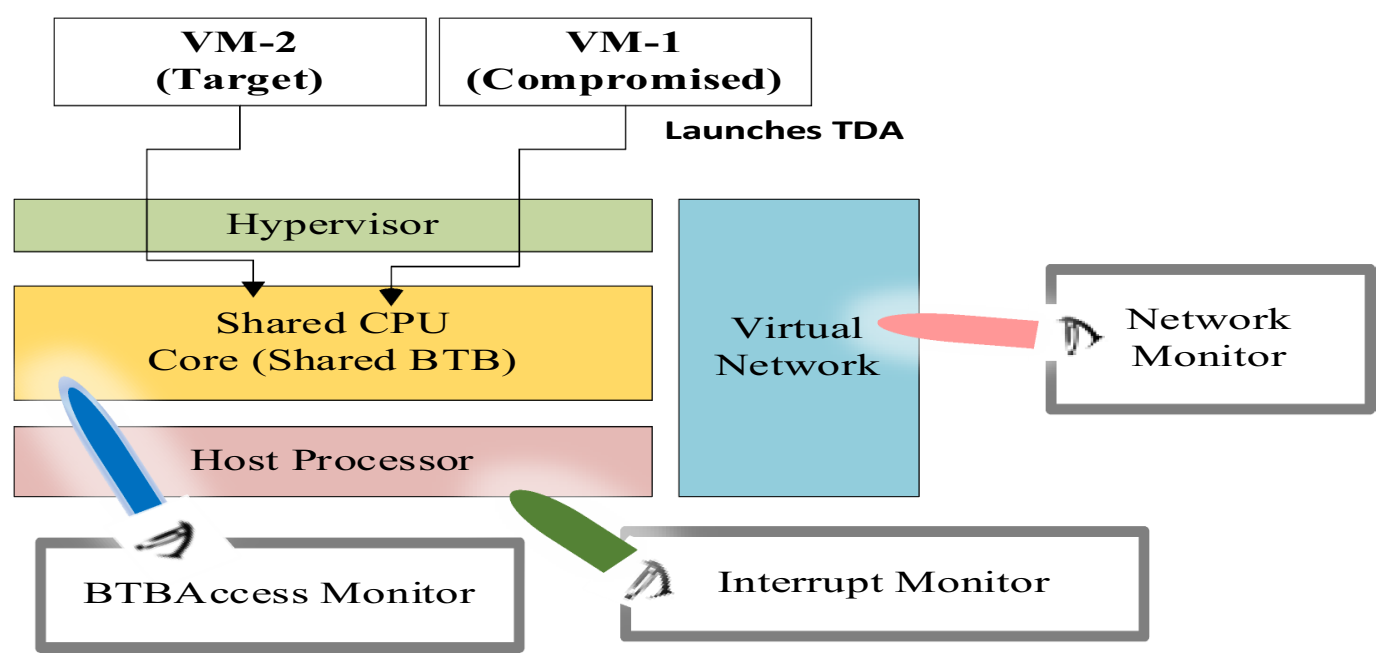

Fig. 3 Model of Trinetra 


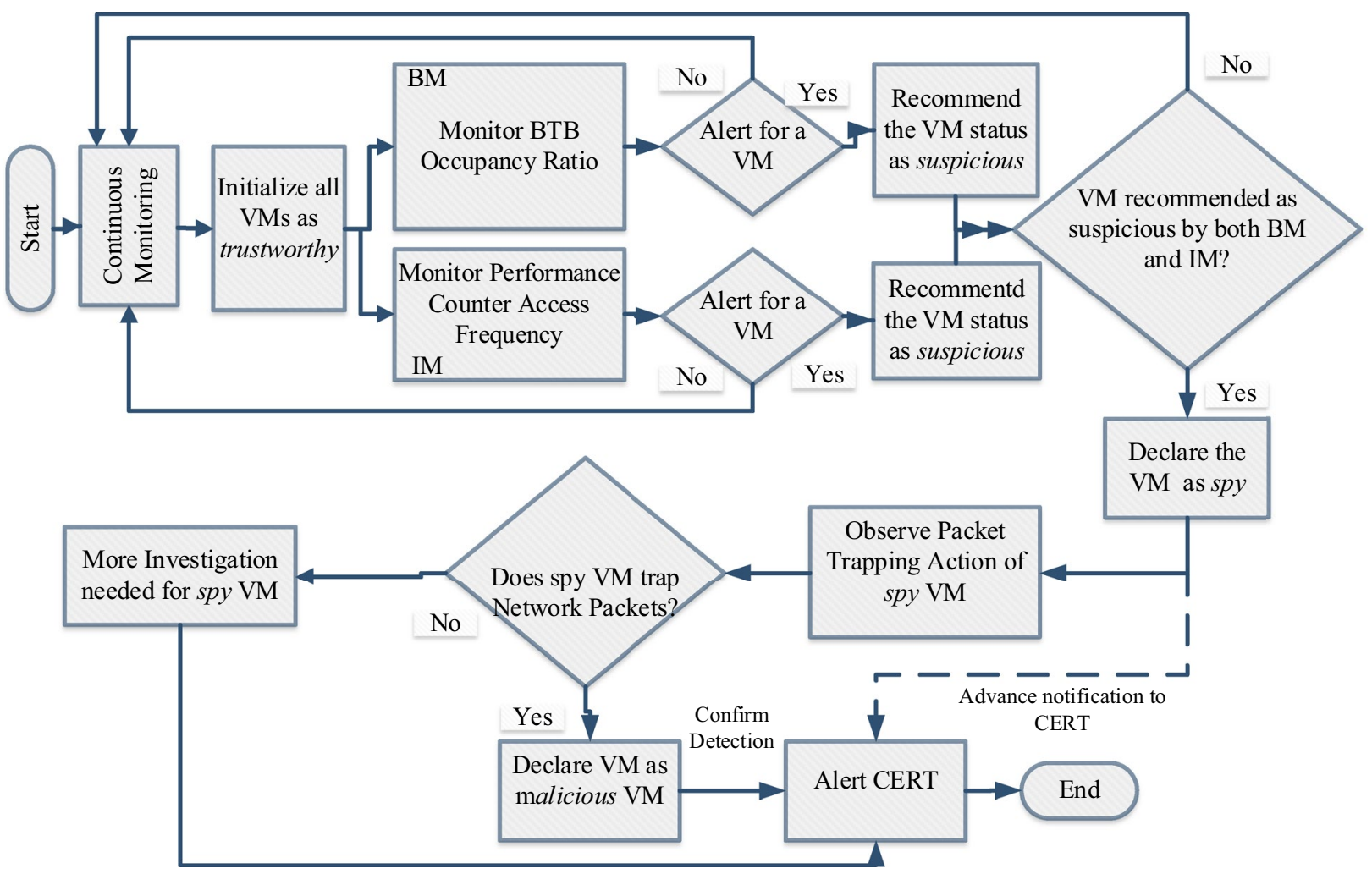

Fig. 4 Flow of Trinetra

instructions. Retrieved number of BTB hits and known size of BTB reveals the BTB occupancy of a process.

From the experimental analysis, we found that the BTB occupancy of the spy process is much more than any other processes. For a set threshold value of the BTB occupancy ratio, BTBAccess monitor generates alert for all the processes resulting in BTB occupancy higher than the threshold value. The value of the threshold point is decided with experimental analysis. Further, the status of trustworthy VM with BTB occupancy ratio crossing the threshold value is recommended to be changed to suspicious.

(b) Interrupt monitor: In concurrence to BM, IM keeps track of the access frequency of the performance counter for all the VMs.

$\mathrm{IM}$ is designed to monitor the access frequency of the hardware performance counters for all the VMs. HPCs can be accessed from any privilege level provided PCE (performance monitoring counter enable) bit of control register CR4 is set. However, the bit is 0 in default case that requires a special privilege to read HPCs.

When access to HPCs is requested, it results in a system call namely, SYS_PERF_EVENT_OPEN. System calls generated while trying to access performance monitoring counters (PMCs), time-stamp coun- ters (TSCs) and machine status registers (MSRs) are trapped into the respective process defined in $m s r . h$ file of the Linux kernel. In the arch directory of kernel source, we followed $\times 86$ directory as per the underlying architecture of the system under consideration. Within $\times 86$ directory, include/asm/msr.h was the path of the file containing native_read_pmc() function. System call generated during HPC reading, i.e., SYS_PERF_EVENT_OPEN is trapped in this native read_pmc of the msr.h file.

Implementation of IM is done by adding code in this Linux kernel function native_read_pmc() of the Ubuntu [19] operating system. Code is added to this function to count the total number of times the HPCs are accessed by each of the running processes. Like $\mathrm{BM}$, IM sets a threshold for the counter, keeping track of HPC access frequency. An alert is generated for the VM for which HPC access frequency crosses the threshold value. The threshold value is decided after observing the results by setting it to different numbers as discussed in Sect. 7. Status of the VM for which alert is generated is recommended to suspicious.

If the status of a VM is recommended to be changed from trustworthy to suspicious by BTBAccess monitor as well as by the interrupt monitor, then the status of 
that VM is changed to spy. Further, network monitor is started for the spy VM.

(c) Network monitor: Monitoring of packet trapping activity of the spy VM is the primary function of NM. However, the way of trapping packets depends on the applications. Data of FTPS and HTTPS can be trapped over the network as well as from the disk, while the data of a secure video conferencing can be trapped only over the network. Detection of such activity can be carried out by manual, automatic and semiautomatic methods. Among them, the semiautomatic model is the most popular as the manual methods are difficult to implement and the automatic methods are resource hungry. The semiautomatic method waits for a trigger from external ways and means to start the required automatic method. The third eye of our architecture, NM, helps the security team to trigger for their semiautomatic method activated.

We have launched the ARPspoofing method to trap packets and have used the ARP spoofing detection module as a plug-in in the code of NM to prove its effectiveness. However, the security team of the system can replace it with the semiautomatic method in use as shown in Fig. 5.

If the network monitor module finds multiple entries of the spy VM, then the spy VM has certainly performed ARPspoofing, and it is declared as malicious. It will raise alert to the computer emergency response team (CERT) about malicious activities for preventing leakage of secured data.

Trinetra is implemented at the host level. There hostbased attacks like VM escape and host-based rootkits that may exploit the host-based module. However, the code of Trinetra is incorporated in the kernel source code. For overwriting or eliminating the Trinetra code, it is needed to recompile the kernel which needs to reboot the system. Sudden system reboot without any means immediately generates alert to CERT. In this way, we can say that the Trinetra is difficult to be compromised. Trinetra does not apply an architectural or functional change, and hence, it does not adversely affect the normal functionality of the

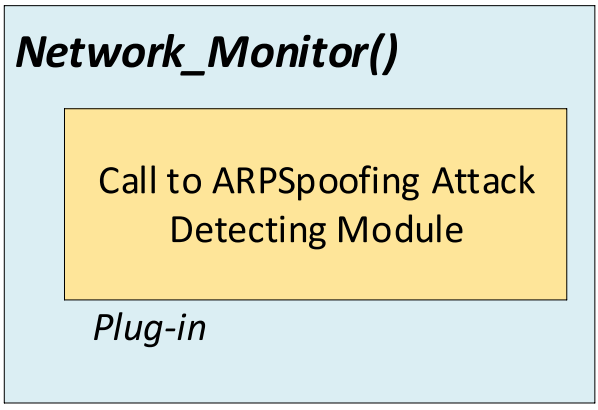

Fig. 5 NM implementation system, and so, the performance of the other processes does not get affected.

False positive is one of the very significant parameters while analyzing the performance of an approach. In the initial phase, we implemented BM and IM of Trinetra to observe the results they obtain for legitimate processes. A detailed discussion on these results is carried out in Sect. 7.

\section{Working out threshold}

Implementation of BM and IM was initiated mainly to identify the threshold values. The simulation of BM was carried out with Gem5 [20] simulator where the output file provides details of the architectural parameter like the number of branches and BTB hits. BTB occupancy ratio of different MiBench [21] programs was calculated for around 1000 times. The plot in Fig. 6 represents the minimum, average and maximum of the observed ratios. The plot reveals that among all the MiBench programs, jpeg has the highest average occupancy which is 0.4. Accordingly, the threshold value for the BM was set to 0.4 .

In concurrence to the BTBAccess monitor, interrupt monitor keeps a continuous watch on performance counter access frequency. The code added to the kernel function native_read_pmc() makes a kernel log when the counter for a process hits the predefined threshold. Initially, the threshold was set to value 10. Experimental analysis was initially carried out on sample programs of MiBench, but we did not find kernel logs for any of the programs for threshold value 10 . However, we found logs for other Linux processes running in the foreground/background, and so, results were collected for them for analysis. For threshold

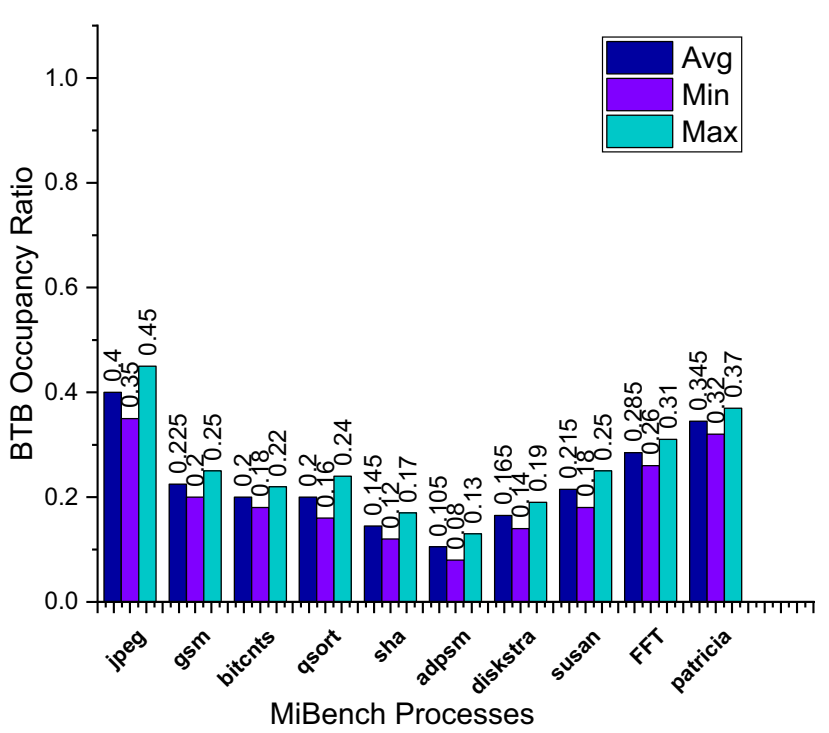

Fig. 6 BTB occupancy ratio observed by BTBAccess monitor 
value 10, we found repeated kernel logs for some of the running processes where the time difference between two successive logs was considered as a parameter for plotting the results. Figure 7 plots the results where the $x$-axis represents the time stamp in seconds when a process hits the threshold value and the corresponding value in the $y$-axis represents the exponential cumulative frequency with which the HPCs are accessed by various running processes. Frequency is calculated by taking the inverse of the time difference between the last two successive logs. As shown in the diagram, we found logs for various benign activities like software updater, Web content and Xorg. The results reveal that the exponential cumulative frequency is between 0.01 and 1 for benign Linux processes. We carried out the experiment for around 1000 times, and we

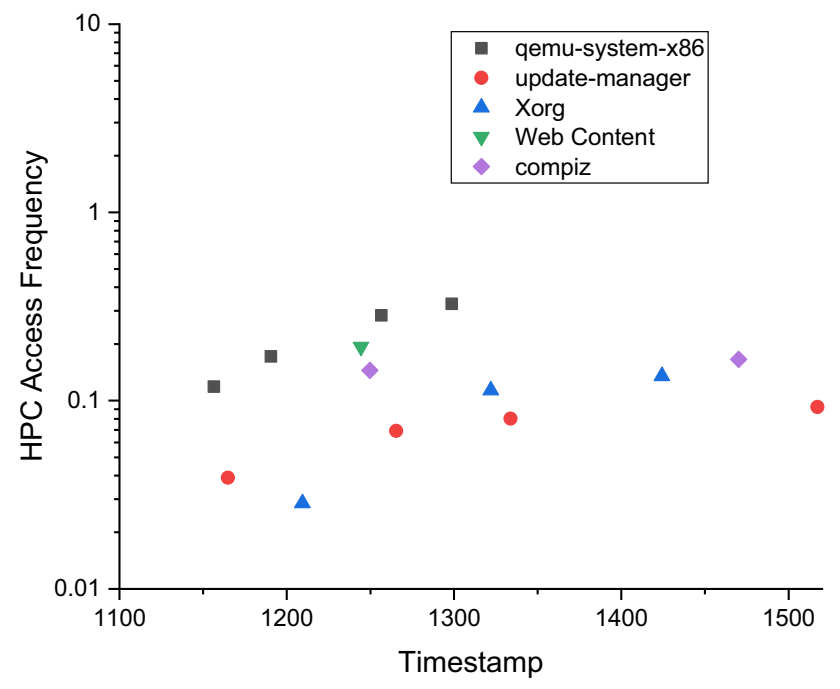

Fig. 7 Observation by IM for counter threshold 10 obtained similar results for all the runs. Accordingly, we found that 1 can be set as the threshold value for the interrupt monitor.

Further, experiments were done for IM even by taking the counter values 20,30 and 40 . We observed from the obtained results that as the counter threshold value was increased, the number of processes generating kernel log was decreased. Moreover, we did not find any kernel log for any of the processes when the counter threshold was set to value 40 . Figure 8 summarizes the above discussion in graphical form.

From the experimental results, we can summarize that there are two ways to set the threshold value for the interrupt monitor: (1) on finding the cumulative HPC access frequency of a process/VM crossing the limit 1 and (2) on finding kernel logs for a process/VM by setting the threshold value 40 .

Experimental analysis of BM and IM was also done by launching the TDA spy process. Discussion on observed results is carried out in Sect. 9, preceded by the discussion on attack simulation in Sect. 8.

\section{Attack simulation}

Simulation of time-driven attack is carried out as a prerequisite for testing the performance of Trinetra. A brief discussion on attack simulation for TDA is provided in this section.

We have used Gem5 [20] simulator for simulating the TDA attack. Gem5 is an open-source multithreaded simulation platform. We created two VMs, VM1 and VM2, on the Gem5 simulator and configured to have common CPU core and so common BTB. Simulation parameters are
Fig. 8 Processes caught by IM for varying counter threshold

\section{Detected Processes for varying Counter Threshold}

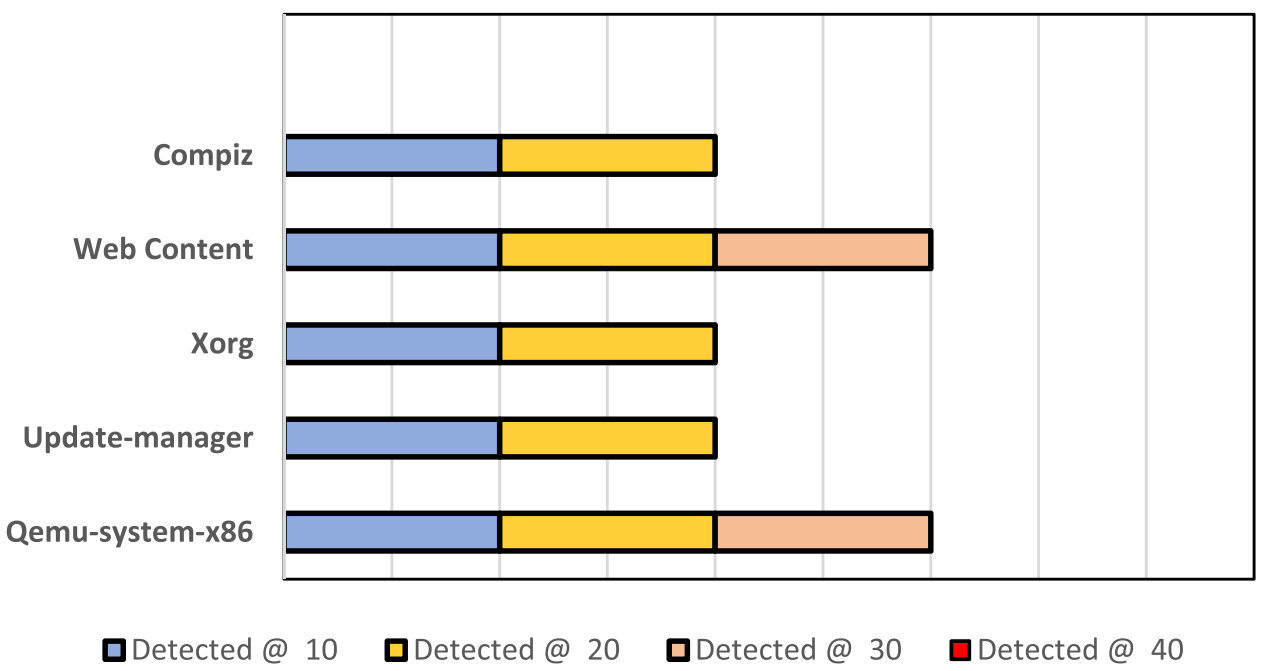


mentioned in Table 2 . The spy process in TDA requires fills the entire BTB which requires a large number of conditional branch instructions. Moreover, the number of conditional branch instructions should also match the total number of BTB entries. To meet the matching criteria discussed previously, we have configured the parameters of Gem5 such that the number of BTB entries matches the total number of conditional branch instructions. Parameters other than BTB entries are left with their default values.

The spy process and RSA cryptographic process were initiated in VM1 and VM2, respectively. Spy process was started before the RSA process starts and it was set to run continuously to observe the execution time as per the attack methodology.

Table 2 Gem5 simulation parameters

Gem simulation parameter with values

BTBEntries $=4096$

BTBTagSize $=16$

globalCtrBits $=2$

globalPredictorSize $=8192$

Max Threads of SMT $=4$

localHisoryTableSize $=2048$

localPredictorSize $=2048$

localCtrBits $=2$
The plot in Fig. 9 represents how bits are predicted (bit value is extracted from the observed execution time as per the TDA procedure) with the TDA procedure. Th number of clock cycles (execution time) of the spy process is the decisive parameter to predict the logic of the private key bit of the RSA decryption algorithm. Figure 9 represents that the observed number of clock cycles is more at those places where the corresponding key bit is 1 in the RSA key. On the opposite side, the number of clock cycles observed by the spy process is comparatively low during the time when the concurrently running RSA process encounters 0 in the key bit. Extracted bit for the range 30-39 are explicitly highlighted for showing the result of the attack launching procedure.

\section{Experiments and results}

Experimental analysis for identifying the threshold values of BM and IM is presented in Sect. 7. Further, we performed the same experiments by launching the TDA spy process. The results observed for BM are shown in Fig. 10. As per Sect. 7, the threshold value for $B M$ was set to 0.4 considering the highest value of BTB occupancy ratio observed in Fig. 6. However, we increase that value to 0.8 for a safer side as per the occupancy ratio observed for the TDA spy process as per Fig. 10. Accordingly, 0.8 is set as the threshold value for generating alert by the IM.

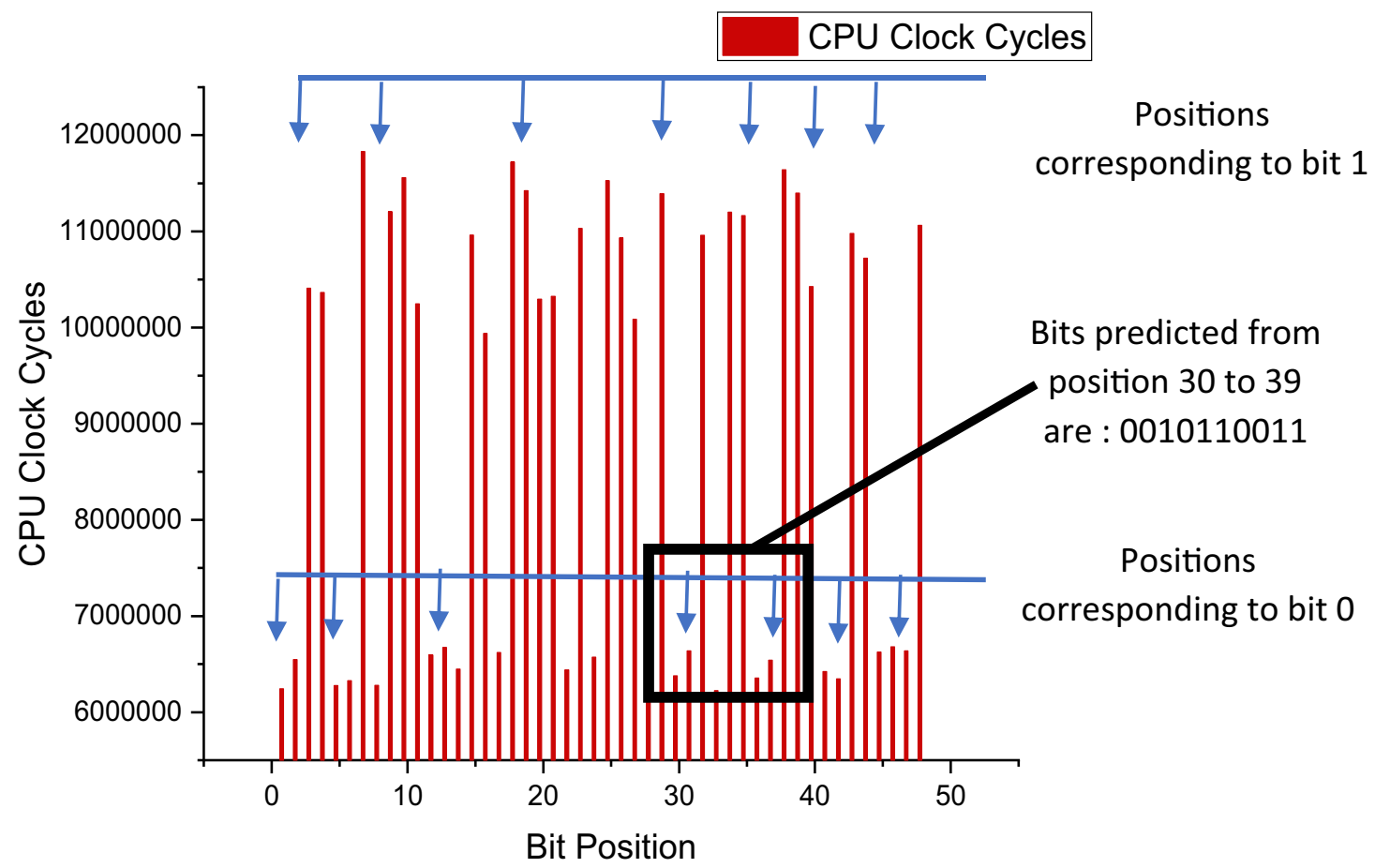

Fig. 9 Results of simulation for time-driven attack 
Fig. 10 BTB occupancy ratio observed by BM (along with spy process)

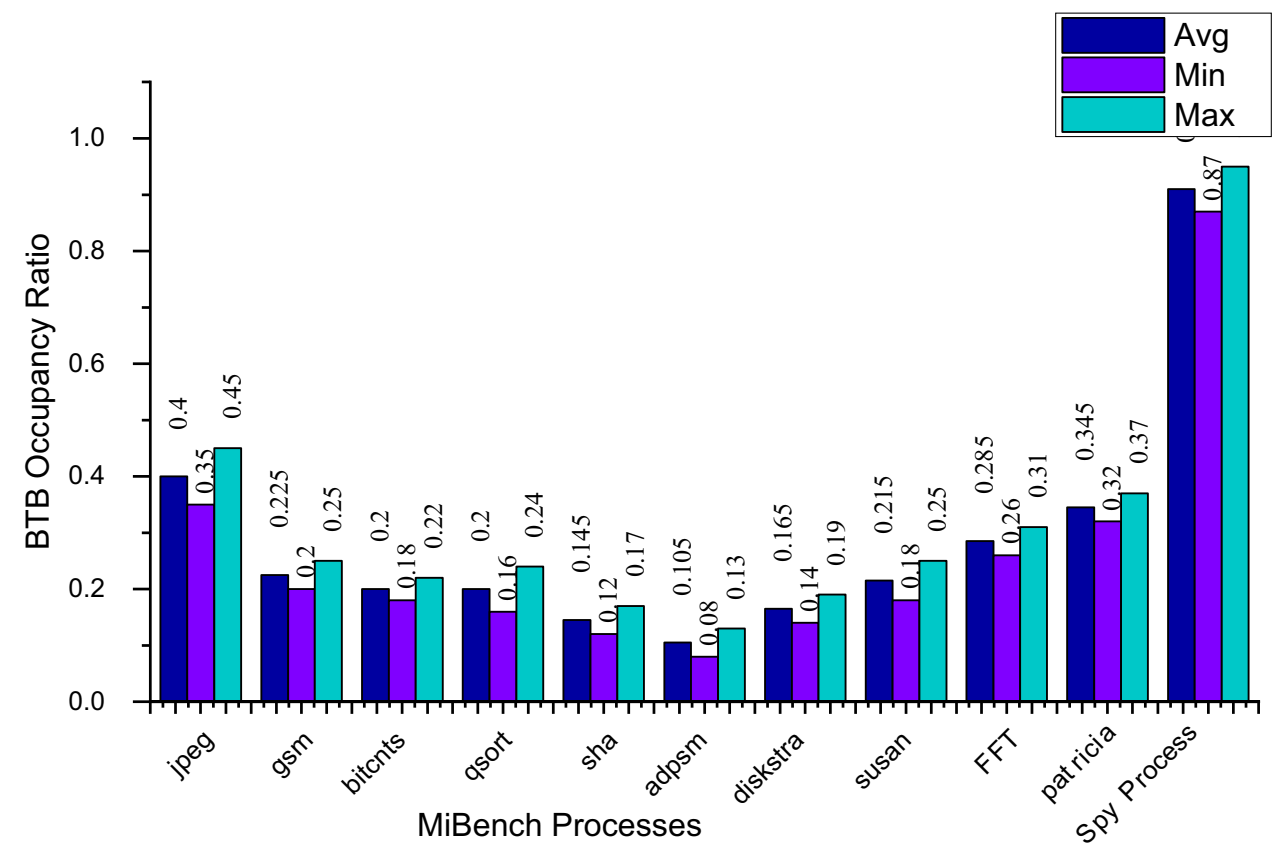

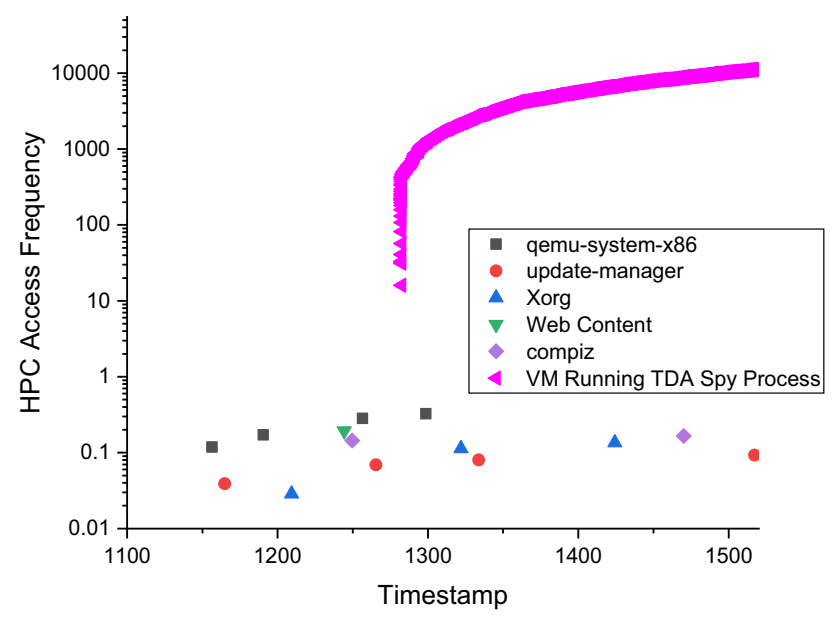

Fig. 11 Observation by IM for counter threshold 10 (along with spy process)

Like BM, the results of IM were also observed in the presence of the VM launching spy process. Obtained results shown in Fig. 11 reveal that where none of the legitimate processes cross the value 1 for the exponential cumulative frequency, the same is found to be more than 100 for the VM running TDA spy process. Although we do not get any false positive when the frequency threshold value is set to 1 based on our initial experiments, we increased it to 100 for a much safer side considering the extremely high access frequency of the TDA spy process.

Additionally, excessive kernel logs were found for that VM running spy process even when the counter was set to value 40 . Figure 12 represents this fact.
By taking the above discussion into account, we revise the threshold criteria for IM for the means of eliminating the scope of false positive. Accordingly, the interrupt monitor can be set to generate an alert: (1) on finding the cumulative HPC access frequency of a process/VM crossing the limit 100 and (2) on finding kernel logs for a process/VM by setting the threshold value 40 . In either of the ways, IM will generate an alert for the TDA spy process within 1-s time period. Additionally, no other legitimate process will be caught by the IM. The process/ VM for which alert is generated by IM is recommended for status change to suspicious.

In turn, the status of VM that reads the performance counter with high frequency and holds high BTB occupancy is recommended to be changed to suspicious by both BTBAccess monitor and interrupt monitor. The status of that VM is further changed to spy.

At this time, the network monitor is launched. Although there may be many ways of trapping network packets, we consider ARPspoofing for our implementation. Accordingly, network monitor targets VM2 and searches for double entries of its MAC address. If the network monitor finds such an entry, then it changes the status of spy VM to malicious and generates alert to CERT for further actions.

Our result analysis reveals that no legitimate process/ VM crossed the set threshold value for any of the two monitors. Moreover, a process/VM is declared as spy only if both BM and IM generate alert for it. In this scenario, even if an alert is generated for a legitimate process/VM by either BM or IM, there are extremely low chances of the process/VM to be caught by both the monitors together. 
Fig. 12 Processes caught by IM for varying counter threshold (along with spy process)
Detected Processes for varying Counter Threshold

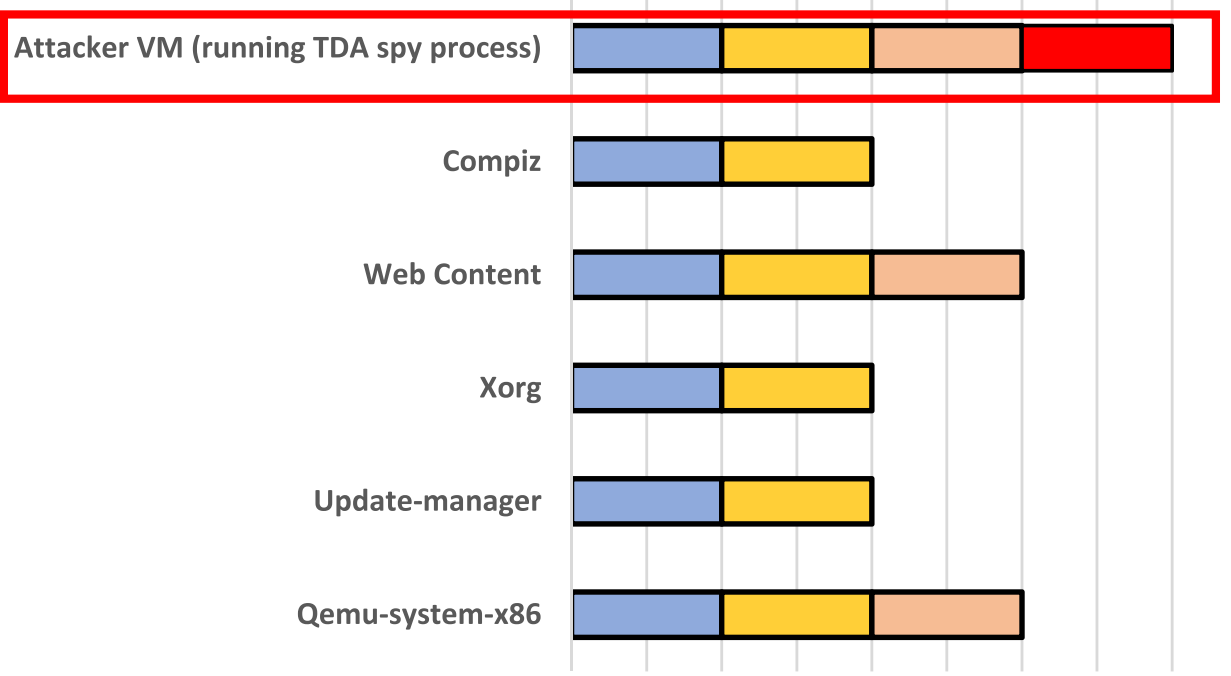

Detected@10
Further, NM is also set as an additional eye to observe whether some packet trapping activity is generated by the attacker. Although packet trapping is not a part of the TDA attack, the attacker needs to carry out it for a fruitful BPA attack as discussed in Sect. 6 . In this regard, if the NM generates an alert for the spy VM, then the process/VM is certainly malicious. Accordingly, we can say that the false positive rate is extremely low in Trinetra. Table 3 presents a comparison of Trilochan with existing solutions.

We evaluated the performance overhead of Trinetra by monitoring the free memory, CPU usage and interrupts encountered in the presence and absence of Trinetra. As per Fig. 13, overhead of Trinetra is within $1 \%$.

\section{Conclusion and future work}

The study of BPA attack in a virtualization environment reveals the necessary resource sharing configuration for launching a cross-VM BPA attack. In this regard, we analyze the scope of existing solutions to handle the crossVM BPA attack. We have identified a need to propose a novel approach to handle BPA attacks that can overcome the limitations of existing solutions. Although the BPA attack can be launched by four different attack methods, we have opted for time-driven attack for our work. We have proposed an algorithm, Trinetra, to handle cross-VM Time-driven attack. Experimental analysis of the proposed approach shows that the Trinetra can detect the presence of the TDA within the duration of $1 \mathrm{~s}$. We have also shown that none of the other benign processes get affected

Table 3 Performance comparison

\begin{tabular}{|c|c|c|c|c|c|}
\hline Approach & $\begin{array}{l}\text { Targets virtualiza- } \\
\text { tion? }\end{array}$ & $\begin{array}{l}\text { Applicable to handle cross- } \\
\text { VM BPA? }\end{array}$ & $\begin{array}{l}\text { Focused BPA mecha- } \\
\text { nism }\end{array}$ & $\begin{array}{l}\text { Affects legitimate } \\
\text { process? }\end{array}$ & $\begin{array}{l}\text { Dependent on crypto- } \\
\text { graphic algorithm? }\end{array}$ \\
\hline Agosta et al. [9] & No & Yes & $\begin{array}{l}\text { Independent of attack } \\
\text { mechanism }\end{array}$ & No & $\begin{array}{l}\text { Yes. It requires } \\
\text { modification in each } \\
\text { vulnerable algorithm }\end{array}$ \\
\hline Tan et al. [10] & No & $\begin{array}{l}\text { Only for VMs with common } \\
\text { core }\end{array}$ & Time-driven attack & Yes & No \\
\hline Julien et al. [11] & Yes & Yes & $\begin{array}{l}\text { Independent of attack } \\
\text { mechanism }\end{array}$ & Yes & No \\
\hline Bhattacharya [12] & No & Yes & Direct timing attack & Yes & No \\
\hline Trinetra & Yes & Yes & Time-driven attack & No & No \\
\hline
\end{tabular}




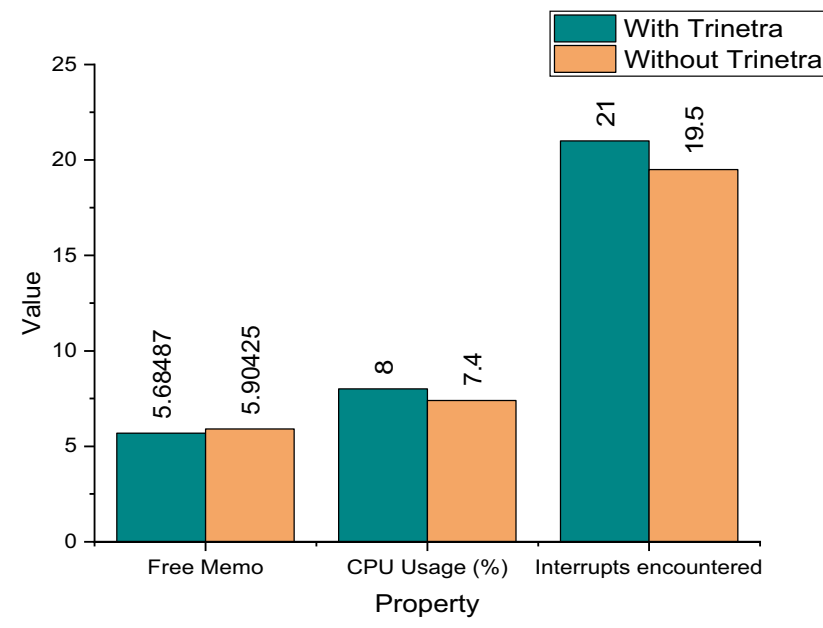

Fig. 13 Performance evaluation in the presence and absence of Trinetra

because of Trinetra, and accordingly, the false positive ratio of Trinetra is almost zero.

Although we target only the TDA method for our approach, we plan to extend the scope of our work by considering all the other attack launching methods also.

Funding This research received no specific grant from any funding agency in the public, commercial or not-for-profit sectors.

Availability of data and material Not applicable.

Code availability Not applicable.

\section{Compliance with ethical standards}

Conflict of interest On behalf of all authors, the corresponding author states that there is no conflict of interest.

\section{References}

1. Betz J, Westhoff D, Müller G (2017) Survey on covert channels in virtual machines and cloud computing. Trans Emerg Telecommun Technol 28(6):3134

2. Seifert J, Koç Ç, Aciiçmez O (2007) Predicting secret keys via branch prediction. In: Ct-Rsa, pp 225-242

3. Ristenpart T, Tromer E, Shacham H, Savage S (2009) Hey, you, get off of my cloud: exploring information leakage in third-party compute clouds. In: Proceedings of the 16th ACM conference on computer and communications security, pp 199-212

4. Aciiçmez O, Koç ÇK, Seifert J-P (2007) On the power of simple branch prediction analysis. In: Proceedings of the 2nd ACM symposium on information, computer and communications security, pp 312-320
5. Zhang Y, Juels A, Reiter MK, Ristenpart T (2012) Cross-VM side channels and their use to extract private keys. In: Proceedings of the 2012 ACM conference on computer and communications security-CCS'12, p 305

6. Vateva-Gurova T, Luna J, Pellegrino G, Suri N (2014) Towards a framework for assessing the feasibility of side-channel attacks in virtualized environments. In: 2014 11th international conference on security and cryptography (SECRYPT), pp 113-124

7. Wang Z, Yang R, Fu X, Du X, Luo B (2016) A shared memory based cross-VM side channel attacks in laaS cloud. In: 2016 IEEE conference on computer communications workshops (INFOCOM WKSHPS), pp 181-186.

8. Buch DH, Bhatt HS (2019) Cross-VM branch prediction analysis attack : scope assessment and simulation. Int J Recent Technol Eng 2:4868-4873

9. Agosta G, Breveglieri L, Pelosi G, Koren I (2007) Countermeasures against branch target buffer attacks. In: Workshop on fault diagnosis and tolerance in cryptography (FDTC 2007), pp 75-79.

10. Tan Y, Wei J, Guo W (2015) The micro-architectural support countermeasures against the branch prediction analysis attack. In: Proceedings of the 2014 IEEE 13th international conference on trust, security and privacy in computing and communications, pp 276-283

11. Sebot J, Gueron S (2014) Mitigating branch prediction and other timing based side channel attacks. US Pat 8869294

12. Bhattacharya S, Bhasin S, Mukhopadhyay D (2018) Online detection and reactive countermeasure for leakage from BPU using TVLA. In: 2018 31st international conference on VLSI design and 2018 17th international conference on embedded systems (VLSID), 2018, pp 155-160

13. Buch $D$, Bhatt $H$ (2018) Taxonomy on cloud computing security issues at virtualization layer. Int J Adv Res Eng Technol (IJARET) 9(4):50-76

14. Joye M, Yen S-M (2002) The montgomery powering ladder. In: International workshop on cryptographic hardware and embedded systems (CHES 2002), pp 291-302

15. Bhattacharya $S$, et al (2014) Fault attack revealing secret keys of exponentiation algorithms from branch prediction misses. In: IACE Cryptogr. ePrint, p 790

16. Bhattacharya S, Mukhopadhyay D (2015) Who watches the watchmen? Utilizing performance monitors for compromising keys of RSA on intel platforms. Springer, Berlin, pp 248-266.

17. Bhattacharya S, Mukhopadhyay D (2017) Formal fault analysis of branch predictors: attacking countermeasures of asymmetric key ciphers. J Cryptogr Eng 7(4):299-310

18. Kulah Y, Dincer B, Yilmaz C, Savas E (2019) SpyDetector: an approach for detecting side-channel attacks at runtime. Int J Inf Secur 18(4):393-422

19. The leading operating system for PCs, loT devices, servers and the cloud|Ubuntu. [Online]. https://ubuntu.com/. Accessed: 13 Feb 2020

20. Binkert $\mathrm{N}$ et al (2011) The gem5 simulator. ACM SIGARCH Comput Archit News 39(2):1

21. Guthaus MR, Ringenberg JS, Ernst D, Austin TM, Mudge T, Brown RB (2001) MiBench : a free, commercially representative embedded benchmark suite The University of Michigan Electrical Engineering and Computer Science. In: Proceedings of the workload characterization, pp 3-14

Publisher's Note Springer Nature remains neutral with regard to jurisdictional claims in published maps and institutional affiliations. 